\title{
Traduire
}

Revue française de la traduction

\section{La terminologie à la petite semaine : gérer la terminologie dans un petit service}

Natalie Garde

\section{(2) OpenEdition}

Journals

Édition électronique

URL : http://journals.openedition.org/traduire/963

DOI : $10.4000 /$ traduire.963

ISSN : 2272-9992

Éditeur

Société française des traducteurs

Édition imprimée

Date de publication : 1 juin 2008

Pagination : 52-60

ISSN : 0395-773X

\section{Référence électronique}

Natalie Garde, "La terminologie à la petite semaine : gérer la terminologie dans un petit service »,

Traduire [En ligne], 217 | 2008, mis en ligne le 01 juin 2008, consulté le 01 mai 2019. URL : http:// journals.openedition.org/traduire/963; DOI : 10.4000/traduire.963 


\title{
La terminologie à la petite semaine : gérer la terminologie dans un petit service
}

\author{
Natalie Garde
}

\section{Traductrice au CERN}

La question de la terminologie dans un petit service de traduction est forcément épineuse. Si la taille du service ne justifie pas l'existence d'une unité spécialisée de terminologie, il apparaît néanmoins nécessaire de gérer les ressources terminologiques. C'est ainsi que les traducteurs sont amenés à faire eux-mêmes un travail de terminologie. Or, nous le savons tous, le recensement terminologique peut être très utile à long terme, mais il n'est jamais urgent, contrairement à ce communiqué de presse qui ne sera plus d'actualité dans un mois, mais qui trépigne d'impatience sur mon bureau. Il faudra donc essayer d'intégrer un travail de terminologie, mené méthodiquement si possible, dans l'activité courante du traducteur. Il faudra aussi, tout en respectant les règles de l'art, s'adapter à une situation réelle et à des besoins concrets. J'ai l'intention d'évoquer ici un projet terminologique commencé il y a deux ans dans le service Traduction et procèsverbaux du CERN(1). Le projet consiste, à partir d'une base existante, à construire une base plus étoffée, actualisée de façon plus régulière, avec la participation de tous les traducteurs. La gestion du projet a été confiée à une équipe de terminologie comprenant à ce jour deux personnes.

(1) À l'origine, l'acronyme correspondait à "Conseil européen pour la Recherche nucléaire ", un organe provisoire institué en 1952, qui avait pour mandat de créer en Europe une organisation de rang mondial pour la recherche en physique fondamentale.

Lorsque le Laboratoire vit officiellement le jour, en 1954, le Conseil provisoire fut dissous et la nouvelle organisation fut baptisée « Organisation européenne pour la Recherche nucléaire ». L'acronyme CERN fut toutefois conservé. 
Le CERN est une organisation internationale comptant deux langues officielles, l'anglais et le français. En tant qu'organisation internationale, elle produit, souvent dans les deux langues, un certain nombre de documents administratifs, juridiques et financiers, et notamment des documents destinés à être examinés par les délégations des États membres lors des réunions des organes directeurs. Mais le CERN est aussi, et principalement, un laboratoire scientifique, travaillant dans un domaine hautement spécialisé - la physique des particules - et où se réalisent des projets de haute technologie dans des domaines tout aussi spécialisés (génie civil, cryogénie, techniques du vide, supraconductivité, pour ne citer que quelques exemples). Les registres de langues sont assez divers, puisque les textes produits sont aussi bien des publications assez informelles pour le Bulletin du personnel, des documents de vulgarisation destinés au grand public ou des spécifications techniques pour les entreprises que des documents de conférence officiels à l'intention des États membres et des documents juridiques. Les tâches de traduction et de rédaction des procès-verbaux de réunions sont confiées à un petit service de sept traducteurs (quatre anglophones et trois francophones).

Qu'en est-il de la terminologie ? Le service ne dispose pas d'une unité de terminologie et de référencement, mais les traducteurs ont toujours mené des travaux de terminologie, qui, dans des temps anciens, se concrétisaient par des fiches cartonnées manuscrites et, depuis une quinzaine d'années, par des contributions à plusieurs types successifs de bases de données. Au moment où notre projet démarre, il existe une base constituée de toutes les fiches créées au fil des ans par les traducteurs, sans exigence de forme trop stricte. Les termes ont été systématiquement validés par un réviseur. Il ne faut naturellement pas oublier que, il n'y a pas si longtemps, les traducteurs ne disposaient ni d'une vaste base technique telle que Termium, ni des inépuisables ressources du Web, et que, pour effectuer des recherches documentaires, ils étaient limités aux documents papier. Étant donné la difficulté matérielle de retrouver un terme dans un document de référence, il était alors tout à fait utile de disposer même de simples propositions de tra- 
duction, qui pouvaient donner une piste. À présent, les traducteurs ont à leur disposition différents outils permettant de retrouver sans trop de difficultés les travaux précédents, ainsi qu'une mémoire de traduction, qui donne accès très facilement à des paires de phrases (langue source-langue cible) contenant le terme recherché. Avec le temps, ils se sont habitués à consulter essentiellement la mémoire, en négligeant peut-être de consulter et aussi d'alimenter la base de données terminologique.

La première question a donc été de savoir s'il était utile d'avoir une activité de terminologie. S'il s'agit simplement de retrouver une « traduction possible " (un terme utilisé dans un contexte semblable pour un travail antérieur), la mémoire de traduction suffit. S’il s'agit de retrouver le terme utilisé dans un document de référence, en théorie, cela peut se faire assez facilement par une recherche automatique dans les versions électroniques. Or, si la base terminologique du service n'est pas beaucoup consultée, c'est sans doute pour deux raisons : d'abord, on n'y trouve pas tout - faute de ressources, faute de temps, la base n'a pas été suffisamment développée ; d'autre part, souvent, la fiche ne contient guère plus qu'une proposition de traduction, sans autres éléments - exactement ce qu'on trouve aussi dans la mémoire de traduction, et moins que ce qu'on trouve au moyen des outils de référence. Pour que le travail de terminologie soit utile, il faut donc qu'il apporte quelque chose de plus.

Dans le projet de nouvelle base, il a été décidé de suivre des principes plus rigoureux pour la constitution des fiches et de s'efforcer de donner les éléments utiles sur chaque terme traité. L'idée est que, pour chaque terme, et dans les deux langues si la fiche est bilingue, ce qui est généralement le cas, on ait une indication de source claire et précise, des renseignements sur l'utilisation du terme s'il y a lieu, et une validation par un réviseur. Un autre principe est que la présentation de la fiche doit être normalisée : présentation des informations dans un certain ordre, indication des sources selon un modèle bien défini, règles typographiques à respecter. Cette normalisation constitue une contrainte pour l'auteur de la fiche, mais le but est d'avoir à la fin un ensemble 
cohérent, qui sera plus lisible pour les utilisateurs. Rien que de très normal s'agissant d'une base de terminologie.

Étant donné le temps nécessaire à l'établissement d'une fiche selon ces principes, on peut se demander quel est l'intérêt d'effectuer ce travail - et la question a effectivement été débattue. Qu'apporte au traducteur la fiche terminologique par rapport à l'exemple de traduction trouvé au hasard dans la mémoire de traduction ou les archives ?

Tout d'abord, le terme répertorié sur la fiche est - en tout cas il est censé être - fiable, plus fiable que la simple reprise d'une traduction précédente. Il arrive assez régulièrement, en effet, qu'on découvre à l'occasion de recherches un peu plus approfondies que tel terme, utilisé précédemment et repris dans des traductions successives, n'est pas tout à fait correct. Ce qui fait la fiabilité d'une fiche, c'est d'abord la qualité de la source, ce qui nous a amenés à réfléchir à ce qui constitue ou non une source fiable, autrement dit, quel type de document fautil privilégier? (Étant entendu que dans les cas désespérés on fait feu de tout bois). Ainsi, s'il s'agit du nom d'un comité, on prendra comme source le document instituant ce comité et définissant son mandat. $S^{\prime}$ il s'agit d'un terme technique, on prendra une source considérée comme valable - par exemple, pour un terme scientifique, les publications d'un grand laboratoire. À noter que les sources ne se limitent pas aux documents ; le terme peut aussi être proposé par un expert du domaine. La source, systématiquement indiquée, est en soi une information utile. Par ailleurs, pour assurer la fiabilité du point de vue linguistique, le terme est validé par un réviseur expérimenté.

D'autre part, le traducteur trouve dans la fiche des renseignements complémentaires très utiles, concernant l'usage du terme (par exemple, " appellation antérieure à 2003 ", ou " terme propre à l'administration fédérale suisse "), ou des renvois à des termes connexes (par exemple, la fiche "appel d'offres" renvoie à la fiche "étude de marché " car il s'agit de deux éléments d'une même procédure), parfois des explications d'ordre documentaire (toujours assorties d'une source). 
Autre avantage, la terminologie du service est mieux harmonisée. Certes, en principe, les traducteurs n'inventent pas les termes, sauf peut-être pour parler d'un objet nouveau. Ils les reprennent de bonne source et, comme la plupart des traductions sont relues, cela assure une certaine cohérence. Mais dans la pratique il y a toujours des divergences, parce qu'on ne consulte pas toujours les mêmes sources, parce qu'on juge différemment de ce qu'est une "bonne " source, parce que différents types de documents appellent des registres différents, ou parce qu'on a été trop pressé par le temps. La fiche indique quel est le terme consacré au sein du service, ce qui n'empêche pas de répertorier, à des fins d'information, des termes concurrents pour la même notion. Ainsi, un certain type de détecteur de particules est appelé parfois " détecteur de traces " et parfois " trajectographe ". Dans la section française du service Traduction du CERN, on emploie exclusivement le deuxième terme ; il n'est cependant pas inutile de savoir que l'autre terme est également employé par des auteurs francophones. Le service est parfois amené aussi à forger des termes (par exemple pour des organes ou des procédures cités dans des documents de l'Organisation et qui n'existent qu'en anglais). La fiche indique, dans ce cas, " traduction non officielle proposée par le service ». La base permet de garder une trace d'un travail de normalisation linguistique qui s'effectue de toute façon au sein du service.

Enfin, un autre avantage de la base, non négligeable en ces temps où chacun se soucie de développement durable, est qu'elle permet de recycler utilement toutes les recherches que le traducteur est amené à faire pour les besoins de sa traduction. Qui parmi vous ne passe pas souvent une demi-heure à chercher un terme, à fouiller dans les directives européennes ou dans des catalogues de produits techniques, afin de comprendre de quoi il s'agit et de trouver l'appellation correcte ? Si le traducteur se contente d'utiliser ce terme, sans intégrer dans la base les renseignements qu'il a recueillis, son effort est presque entièrement perdu. Il est en tout cas perdu pour ses collègues, et c'est fort dommage. L'existence d'une base terminologique permet de rentabiliser la recherche qu'effectue de toute façon le traducteur. 
Nous avons dû définir un certain nombre de règles de forme pour les fiches - règles qui ont parfois évolué devant certaines contraintes et ont été discutées au sein du service. Comment indiquer une référence qui est en fait un document trouvé sur le web, dans la mesure où les adresses web sont peu parlantes et trop éphémères ? Peut-on indiquer directement sur la fiche le nom de l'expert consulté ? (Nous avons décidé que non.)

Pour construire la nouvelle base, nous disposions de plusieurs possibilités : réutiliser les éléments présents dans l'ancienne base, procéder à un recensement terminologique systématique à partir de documents importants - par exemple, les Statut et Règlement du personnel, la Convention constitutive du CERN, le Règlement financier - et enfin, procéder de façon " opportuniste ", c'est-à-dire établir des fiches pour tous les termes ayant nécessité des recherches approfondies pour une traduction ou la rédaction d'un procès-verbal.

Finalement, nous avons conclu que nous ne disposions pas des ressources nécessaires pour un travail systématique et avons décidé de travailler selon deux axes : d'une part, le transfert d'une partie des données de l'ancienne base, et d'autre part la création de fiches au fil des traductions.

La première étape a consisté à examiner les fiches de l'ancienne base, ce qui était d'ailleurs assez amusant. J'ai pu constater - puisque les fiches sont signées, même si les auteurs sont parfois des inconnus pour moi - que les fiches reflètent la personnalité de celui qui les crée : plus ou moins impulsif, plus ou moins méthodique, plus ou moins expérimenté ; certains font des fiches pour tout, d'autres se limitent aux termes les plus ésotériques... Une partie de cette ancienne base provient de recensements systématiques, ce qui se voit aussi à la lecture de la fiche.

Nous avons décidé de nous limiter, pour le transfert des anciennes fiches, aux termes particulièrement importants dans le travail de l'Organisation - les noms d'organes officiels, de machines importantes appartenant à l'histoire du laboratoire, par exemple, et à ceux pour les- 
quels on dispose dans l'ancienne fiche des éléments nécessaires - car dans ce cas le travail demandé est minime. À noter au passage qu'il est bien utile d'avoir un répertoire des organes, des services et des machines qui existaient il y a vingt ans. Quand on traduit par exemple des textes évoquant l'histoire de l'Organisation, cela permet de retrouver plus facilement le nom de choses disparues avant l'invention du web et des mémoires de traduction. Par contre, nous avons décidé de sacrifier toutes les entrées pour lesquelles nous ne disposions de rien d'autre qu'une paire de termes : retrouver des sources et un contexte prendrait beaucoup de temps, et l'utilité d'un tel travail n'est pas évidente pour des termes qui ont été intégrés à la base il y a fort longtemps. Travailler sur les anciennes fiches a d'ailleurs été l'occasion de réfléchir sur ce qui est ou non utilisable, et s'est avéré profitable pour la définition des règles pour la nouvelle base.

Parallèlement, des fiches sont également créées à l'occasion des traductions effectuées. L'avantage de telles fiches est qu'on sait qu'elles répondent à un besoin, et que les recherches ont déjà été effectuées. Reste le travail de mise en forme, qui n'est pas mince et qui peut être fastidieux pour celui qui n'en a pas l'habitude. Néanmoins, il nous paraît important que tous les traducteurs contribuent à alimenter ainsi la base. Si les fiches ne sont créées que par certains membres du service, elles refléteront leurs besoins propres et ne répondront pas forcément aux attentes de tous. En particulier, dans le cas du service Traduction du CERN, les anglophones font plus de rédaction et moins de traduction que les francophones, et, lorsqu'ils traduisent, les textes ne sont pas forcément du même type que les textes traduits vers le français. Pour donner quelques exemples, la section française a plus souvent affaire à des textes parlant de physique, et la section anglaise plus souvent à des textes techniques très détaillés dans le domaine du génie civil ; les groupes de travail et comités ad hoc sont le plus souvent constitués dans des documents originaux en anglais - ce qui n'empêche d'ailleurs pas, dans ce dernier cas, qu'il soit nécessaire de retenir une appellation uniforme... Si l'on évoque les besoins divers au sein de l'équipe, il faut aussi noter des différences d'appréciation. Il 
semble que les francophones soient plus " rigides » dans leur souci de normalisation, et les anglophones plus souples et plus prêts à accepter des variantes. Je ne sais pas s'il faut l'attribuer à des différences culturelles ou simplement au fait que les travaux effectués ne sont pas les mêmes.

Pour toutes les fiches de la nouvelle base, une procédure de validation a été mise en place. Une première validation sur la forme est effectuée : l'un des membres de l'équipe de terminologie vérifie que la fiche répond bien aux critères formels d'indication de source, etc. Puis la fiche est relue par un réviseur de chaque langue, pour vérifier l'aspect linguistique.

Les règles du jeu sont maintenant claires, le circuit de validation est en place et, petit à petit, la base s'étoffe. Comme on peut l'imaginer, la constitution d'une base terminologique prend du temps, et les traducteurs sont la plupart du temps accaparés par d'autres tâches. Il faut aussi lancer la machine ; chacun doit se familiariser avec les règles définies et se souvenir des manipulations à effectuer. Pour l'instant, le travail est à peine commencé ; la tâche devrait dans l'idéal être vraiment intégrée à la vie du service et chacun devrait y consacrer un peu de temps. Temps que, naturellement, nous n'avons pas. Mais petit à petit, on peut espérer que les choses vont se mettre en place. Au fur et à mesure que les notions nouvelles sont (plus ou moins) systématiquement répertoriées, les traducteurs pourront de plus en plus compter sur cette ressource. Lentement mais sûrement, telle doit être notre devise. Ou plutôt, fastidieusement, mais fiablement.

Si vous avez poursuivi votre lecture jusqu' ici, c'est sans doute que vous aussi vous n'êtes pas insensible au charme du travail terminologique. Et je peux donc vous l'avouer : même si je suis convaincue de l'utilité de cette recherche, qui permet de travailler de façon plus rigoureuse et à long terme de gagner du temps, ce n'est là qu'un aspect de la question - celui qu'on peut faire valoir pour justifier le temps passé à alimenter une base. Mais, en vérité, si on a envie de se lancer dans l'entreprise, c'est aussi qu'on éprouve une grande satisfaction (proba- 
blement thérapeutique) à mettre de l'ordre et de la logique dans cette masse de mots qui nous assaillent sans cesse. Confronté tous les jours à des textes qui partent dans tous les sens, à une langue mouvante, à des auteurs qui parfois s'expriment pour des initiés sans craindre d'être imprécis ou elliptiques, le traducteur se donne l'illusion d'une certaine maîtrise en mettant en fiches ces mots insaisissables, un peu comme l'enfant qui classe inlassablement par forme et par couleur les coquillages qu'il a ramassés pendant les vacances, et qui a ainsi le sentiment de faire tenir sur ses étagères tous les abysses sous-marins.

Après avoir débuté dans une entreprise multinationale, Natalie Garde a poursuivi sa carrière dans différentes organisations intergouvernementales. Elle a notamment travaillé pendant une dizaine d'années comme traductrice free lance à Genève, dans des organisations du système des Nations Unies. Elle est à présent traductrice au Service des traductions et procès-verbaux de l'Organisation européenne pour la Recherche nucléaire (CERN), à Genève. Elle est membre de l'Organisation internationale des traducteurs de conférence (AITC). 